\title{
Influence of Vision on Gait Initiation and First Step Kinematics in Young and Older Adults
}

\author{
Jana KIMIJANOVÁ ${ }^{1}$, Zuzana HIRJAKOVÁ ${ }^{1}$, Diana BZDÚŠKOVÁ ${ }^{1}$, František \\ HLAVAČKA ${ }^{1}$
}

${ }^{1}$ Department of Behavioural Neuroscience, Institute of Normal and Pathological Physiology, Centre of Experimental Medicine, Slovak Academy of Sciences, Bratislava, Slovak Republic

Received April 15, 2021

Accepted October 11, 2021

\section{Summary}

The study investigated whether anticipatory postural adjustments (APAs) of gait initiation and kinematics of the first step are modified with absence of vision in relation to age. Twenty-two young and twenty-two older subjects initiated a self-paced gait with the vision available and deprived. APAs were measured by:

(1) force platform and evaluated by maximal amplitude of the center of pressure (CoP) displacements; (2) two inertial sensors attached to the trunk and evaluated by maximal accelerations. Step kinematics was recorded using a motion capture system and evaluated by duration, length and maximal velocity of the first step. Visual deprivation led to a significant reduction of forward trunk accelerations during the anticipatory phase of stepping in older adults. Moreover, they significantly reduced first step length and maximal velocity and prolonged duration of the first step. Contrary, young adults did not respond to absence of vision by significant changes of neither APAs, nor first step kinematics. These findings suggest that gait initiation is strongly associated with increased reliance on vision in older adults. We further indicate that trunk accelerations during the anticipatory phase of stepping may be a more sensitive measure to detect age-related changes of APAs due to absent visual information compared to CoP.

\section{Key words}

Vision • Gait initiation • Anticipatory postural adjustments • Step kinematics • Ageing

\section{Corresponding author}

J. Kimijanová, Department of Behavioural Neuroscience, Institute of Normal and Pathological Physiology, Centre of Experimental Medicine, Slovak Academy of Sciences, Sienkiewiczova 1, 81371 Bratislava, Slovak Republic. E-mail: jana.kimijanova@savba.sk

\section{Introduction}

Gait initiation involves the correct sequencing of movement preparation and movement execution. Specifically, anticipatory postural adjustments (APAs) precede the onset of voluntary movement such as gait initiation (Bouisset and Do 2008). Immediately prior to the stepping, APAs act to accelerate the center of body mass forward and towards the stance foot by moving the center of pressure ( $\mathrm{CoP})$ backward and towards the stepping foot. After the unloading of the stepping leg and the toe off, a single support phase of the gait cycle follows. By initial contact of the stepping foot with the ground, a first step is accomplished (Winter 1995). During walking, the visual system provides essential sensory information to maintain dynamic stability. Vision plays a critical role in adapting the basic postural patterns for various environments and in guidance of goal directed movements (Patla 1997). In healthy adults, visual information determines gait direction, walking speed, cadence, stride length and stance phase duration (Jahn et al. 2001, Rietdyk and Rhea 2006, Saucedo and Yang 2017). The ability to manage the competing demands for propulsion and maintaining equilibrium during walking is compromised by the progressive sensory, neural and motor declines associated with ageing. Age-related proprioceptive and vestibular impairments are associated with increased reliance on visual inputs, with vision itself being affected by the process of ageing (Kanekar and Aruin 2014). Based on the close link between postural control and locomotion it can be assumed that there are age-related differences in the impact of visual deprivation 
on dynamic stability and gait (Hallemans et al. 2009b). An impaired balance control and dynamic stability resulting from the sensorimotor deficits increase the risk of fall which is one of the major problems in elderly (Rubenstein 2006, Terrier and Reynard 2015, CuevasTrisan 2017). The studies which examined the potential effects of visual deprivation on gait have concluded that low vision reduces dynamic stability and increases the dependency on the somatosensory and vestibular systems to control gait stability (Saucedo and Yang 2017, Reynard and Terrier 2015, Iosa et al. 2012, Hallemans et al. 2010). Significant decrease in walking speed, step frequency and step/stride length were observed between eyes open (EO) and eyes closed (EC) conditions in healthy adults (Hallemans et al. 2009a, Iosa et al. 2012). Moreover, differences in whole-body kinematics (Hallemans et al. 2009b) along with reduction of upper body stability (Iosa et al. 2012) occurred when the vision was deprived. The withdrawal of visual information had a direct effect on the stride-to-stride fluctuations in young adults and these fluctuations were dependent on the walking speed (Wuehr et al. 2013). In the study of Saucedo and Yang (2017), dynamic stability during treadmill walking was also influenced by the deprivation of vision, where both young and older adults took a cautious gait pattern during walking with eyes closed, but there were no age-related differences observed in gait stability while EC walking. Cromwell et al. (2002) demonstrated that older adults decreased walking speed, cadence and peak head velocity during EC walking. However all of these studies focused on the steady state gait but none of them paid attention to the transition from standing to walking (i.e. gait initiation) which may be especially challenging because of requirement to maintain equilibrium while a forward propulsive force is generated (Jian et al. 1993). Furthermore only few of them inspected how age influences the effect of visual deprivation on gait kinematics (Saucedo and Yang 2017, Reynard and Terrier 2015, Cromwell et al. 2002). Whether such an effect on stepping differs between young and older adults still remains unexplored. Therefore, the purpose of this study was to investigate the effect of visual deprivation on gait initiation and the performance of the first step in both young and older adults. The specific aims were 1) to determine to what extent the visual deprivation affects the anticipatory postural adjustments (i.e. CoP displacements and accelerations of the upper and lower trunk) and the first step kinematics under both visual conditions (i.e. EO vs. EC); and 2) to examine whether such an effect is dependent on age. We hypothesized that older adults would be more cautious when stepping with eyes closed than when stepping with vision available and this would manifest as a reduction of postural and kinematic parameters of gait initiation. We further hypothesized that, regardless of visual condition, the anticipatory postural adjustments and kinematics of the first step would be compromised in older adults.

\section{Methods}

Twenty-two healthy young and twenty-two healthy older subjects (Table 1) participated in the study. None of the subjects reported orthopedic, cardiovascular, or neurological diseases, they had no history of falls, and reported no pain, numbness, tingling or weakness at the time of testing. Prior to participation, all subjects gave written informed consent in agreement with the Declaration of Helsinki and the Local Science Ethical Committee approved the experimental protocol.

Table 1. Participant characteristics.

\begin{tabular}{lccc}
\hline & Young (22-35 years) & Older (65-83 years) & p-value \\
\hline$n$ & 22 & 22 & \\
Gender $(M / F)$ & $9 / 13$ & $9 / 13$ & $<0.001$ \\
Age (years) & $29.0(3.4)$ & $73.7(5.6)$ & $<0.001$ \\
Height $(\mathrm{m})$ & $1.72(0.07)$ & $1.63(0.09)$ & 0.08 \\
Mass $(\mathrm{kg}$ & $66.5(13.3)$ & $73.7(12.2)$ & 0.73 \\
Foot length $(\mathrm{cm})$ & $25.8(1.7)$ & $25.9(1.6)$ & \\
\hline
\end{tabular}

Mean (standard deviation). 
Postural sway (i.e. CoP displacements) during initial stance and step initiation was measured using custom-made force platform $(0.45 \times 0.45 \mathrm{~m})$ equipped with automatic weight correction (see details in Hirjakova et al. 2017) located at the beginning of a 5-m walkway. Trunk accelerations were recorded by two inertial sensors (MTx, Xsens Technologies, B.V., The Netherlands) with inbuilt $3 \mathrm{D}$ accelerometers ( $\pm 1.7 \mathrm{~g}$ range) attached to the anterior trunk at the level of sternum (Acc ST) and the posterior trunk at the level of the fifth lumbar vertebra (Acc L5). Initial stance position was consistent from trialto-trial by tracing foot outlines on the force plate. The following APAs amplitudes were compared from the CoP displacements and upper and lower trunk accelerations: (1) APAs amplitudes in anterior-posterior (AP) direction, that is (i) CoP AP - peak of backward CoP displacement, (ii) Acc L5 AP - peak of forward lower trunk acceleration, and (iii) Acc ST AP - peak of forward upper trunk acceleration from the baseline; (2) APAs amplitudes in medial-lateral (ML) direction, that is (i) CoP ML - peak of lateral CoP displacement towards the stepping foot, (ii) Acc L5 ML - peak of lower trunk acceleration towards the stance foot, and (iii) Acc ST ML - peak of upper trunk acceleration towards the stance foot from the baseline. The onset of APAs was detected by an automated threshold-based algorithm, with threshold set as twice the SD of signal during the initial, pre-step initiation period of each trial (Mancini et al. 2009). The APAs were considered completed at the time of stepping leg heel-off (Rocchi et al. 2006).

Kinematics of the first step was recorded by an optoelectronic 6-camera motion capture system (BTS Smart DX, Italy) and evaluated by duration, length and maximal velocity of the first step. Length and velocity of the first step were measured from the $3 \mathrm{D}$ trajectory of the markers on the lateral malleolus and fifth metatarsal of the stepping leg. The step onset was defined as the first observable increase (from the baseline) in the vertical position trace of the lateral malleolus followed by an increase in the vertical position trace of the fifth metatarsal, and the end was defined when the vertical position trace of the fifth metatarsal returned to the baseline value. First step length was defined as the root mean square of the 3D-coordinate of the malleolus marker during the first step. First step velocity was calculated as the first step length divided by the first step duration (Mancini et al. 2016) and the maximal velocity achieved during the first step duration was evaluated.

All data were acquired with sampling frequency of $100 \mathrm{~Hz}, \mathrm{CoP}$ data were low-pass filtered at $10 \mathrm{~Hz}$ cut-off frequency, acceleration data were low-pass filtered at $3.5 \mathrm{~Hz}$ cut-off frequency (Mancini et al. 2009). For comparison between different body dimensions and subjects, CoP data were normalized to foot length, and trunk accelerations, length and velocity of the first step were normalized to height by detrending normalization (O'Malley 1996).

Standing on the embedded force platform, subjects were instructed to initiate gait with their dominant leg, which was determined by asking which leg they would use to shoot the ball (Van Melick et al. 2017). After hearing an acoustic tone, participants took 5-6 steps forward. During the visually intact step initiation, participants were instructed to fixate gaze on a point at the end of the walkway. Vision was deprived by closing the eyes before stepping and subjects kept their eyes closed also during step initiation and execution of a few next steps. Five trials of step initiation with eyes open and five trials with eyes closed were acquired and averaged. The order of conditions was randomized across the subjects. Data were evaluated and analyzed with MATLAB (MathWorks, Inc., Natick, USA). The normality of distribution of each variable was examined using the Shapiro-Wilk test, homogeneity of variance was tested by the Levene's test. The analyzed parameters were normally distributed and variances between groups were equal. Therefore repeated measures analyses of variance (ANOVA) with the between-subject (Age: young vs. older) and within-subject (Vision: EO vs. EC) factors were conducted to identify possible influences of visual deprivation or age on gait initiation parameters. The Age by Vision interaction effects were also investigated. Significance was set at $p<0.05$. Post hoc independent $t$-tests were used on all dependent variables to identify age-related differences during EO or EC stepping. Post hoc paired $t$-tests were conducted to detect differences between EO and EC stepping within each age group. To reduce Type I error due to the multiple $t$-test comparisons, the Bonferroni correction was applied. All statistical analyses were performed in SPSS 18.0 (IBM, USA).

\section{Results}

Our results showed significant influence of visual deprivation on step initiation in relation to advancing age. A significant main effect of Vision was detected for APAs amplitudes in AP direction during 
stepping: Acc L5 AP $\left(\mathrm{F}_{(1,42)}=8.261, p<0.01\right)$, and Acc ST AP $\left(\mathrm{F}_{(1,42)}=6.227, p<0.05\right)$. Trunk accelerations in ML direction as well as CoP displacements in both directions were not significantly affected by Vision. ANOVA further revealed, that all evaluated kinematic parameters of the first step were significantly affected by Vision: first step duration $\left(\mathrm{F}_{(1,42)}=8.872, p<0.01\right)$, first step length $\left(\mathrm{F}_{(1,42)}=13.896, p<0.01\right)$, and max. velocity of the first step $\left(\mathrm{F}_{(1,42)}=16.145, p<0.001\right)$. Significant age-related changes were also approved by ANOVA where the main factor Age affected all APAs amplitudes in AP direction: CoP AP $\quad\left(\mathrm{F}_{(1,42)}=12.578, \quad p<0.01\right), \quad$ Acc $\quad$ L5 AP $\left(\mathrm{F}_{(1,42)}=6.101, p<0.05\right)$, and Acc ST AP $\left(\mathrm{F}_{(1,42)}=4.423\right.$, $p<0.05$ ). In ML direction, max. amplitude of CoP $\left(\mathrm{F}_{(1,42)}=9.319, p<0.01\right)$ was significantly affected by Age. Among kinematic parameters, the main factor Age significantly affected the first step length $\left(\mathrm{F}_{(1,42)}=37.550\right.$, $p<0.001)$, and max. velocity of the first step $\left(\mathrm{F}_{(1,42)}=20.848, p<0.001\right)$. A significant Age by Vision interaction effect was detected for APAs amplitudes: Acc L5 AP $\left(\mathrm{F}_{(1,42)}=11.651, p<0.01\right)$, and Acc ST AP
$\left(\mathrm{F}_{(1,42)}=4.436, p<0.05\right)$.

Consecutive post hoc analyses showed that visual deprivation did not result in significant changes of neither APAs amplitudes (Fig. 1a-c) nor kinematics of the first step (Fig. 1d-f) in young adults. Contrary, significant differences in forward accelerations of upper and lower trunk (Fig. 1b, c) as well as duration, length and max. velocity of the first step (Fig. 1d-f) were found in older adults during gait initiation with eyes open compared to eyes closed. While initiating the step with eyes closed, older adults significantly reduced forward acceleration peaks of upper and lower trunk (Fig. 1b, c). They also reduced backward peak of $\mathrm{CoP}$ displacement during EC stepping (Fig. 1a), however not significantly. For illustration, excursions of CoP, Acc L5 and Acc ST in AP direction in representative young and older subjects during EO and EC stepping are presented in Fig. 2. Post hoc paired comparisons also revealed that older adults executed significantly shorter and slower first step with longer duration when visual information was withdrawn (Fig. 1d-f).
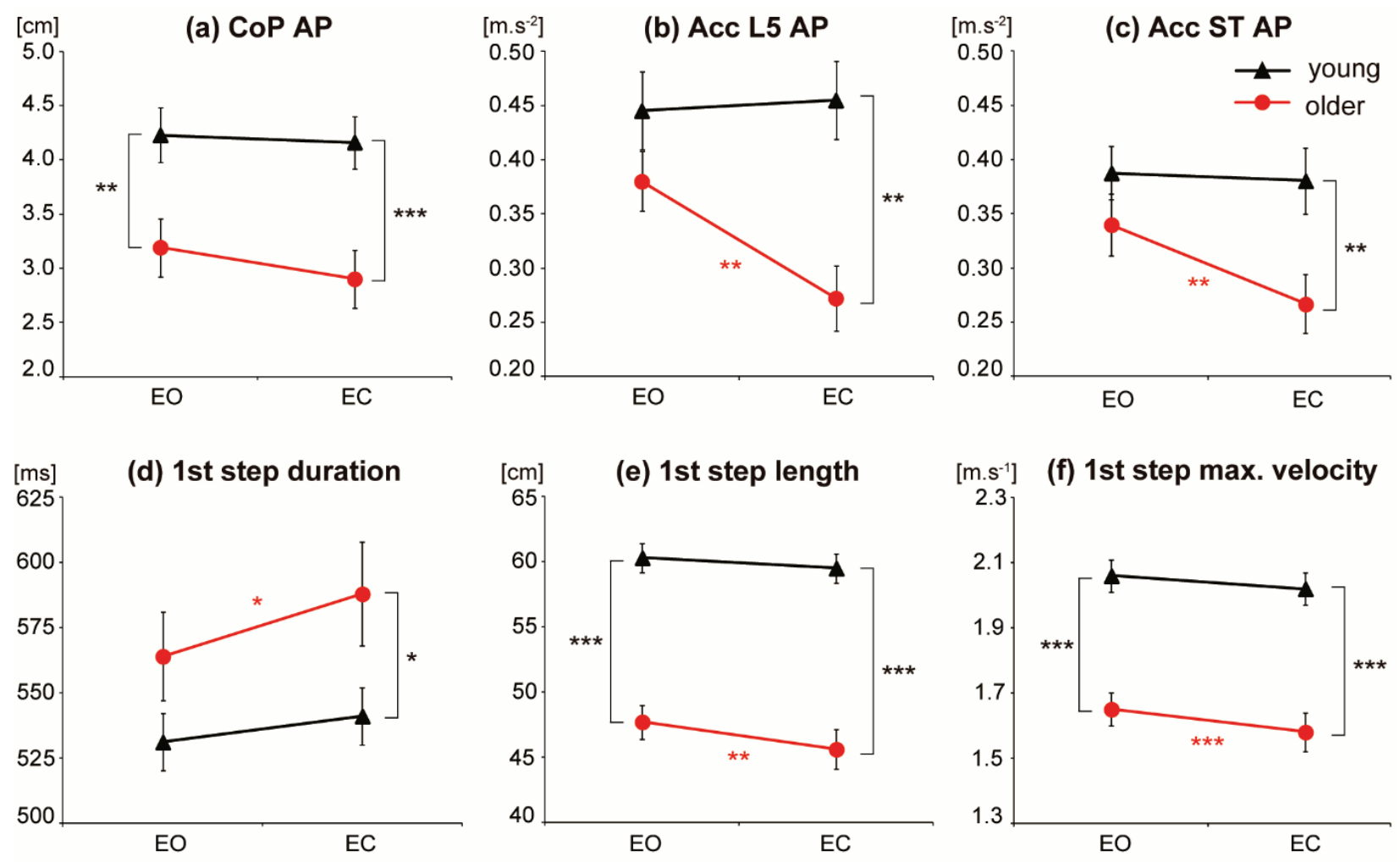

Fig. 1. Comparison of APAs amplitudes: (a) peak of backward CoP displacement, (b) peak of forward lower trunk acceleration, (c) peak of forward upper trunk acceleration; and kinematic parameters of the first step: (d) duration, (e) length, (f) max. velocity for young (black) and older (red) subjects during stepping with eyes open (EO) and eyes closed (EC). Data are presented as group means, error bars represent standard error of the mean. Significant differences between visual conditions EO vs. EC, and between age groups young vs. older are marked: $* p<0.05, * * p<0.01, * * * p<0.001$. 


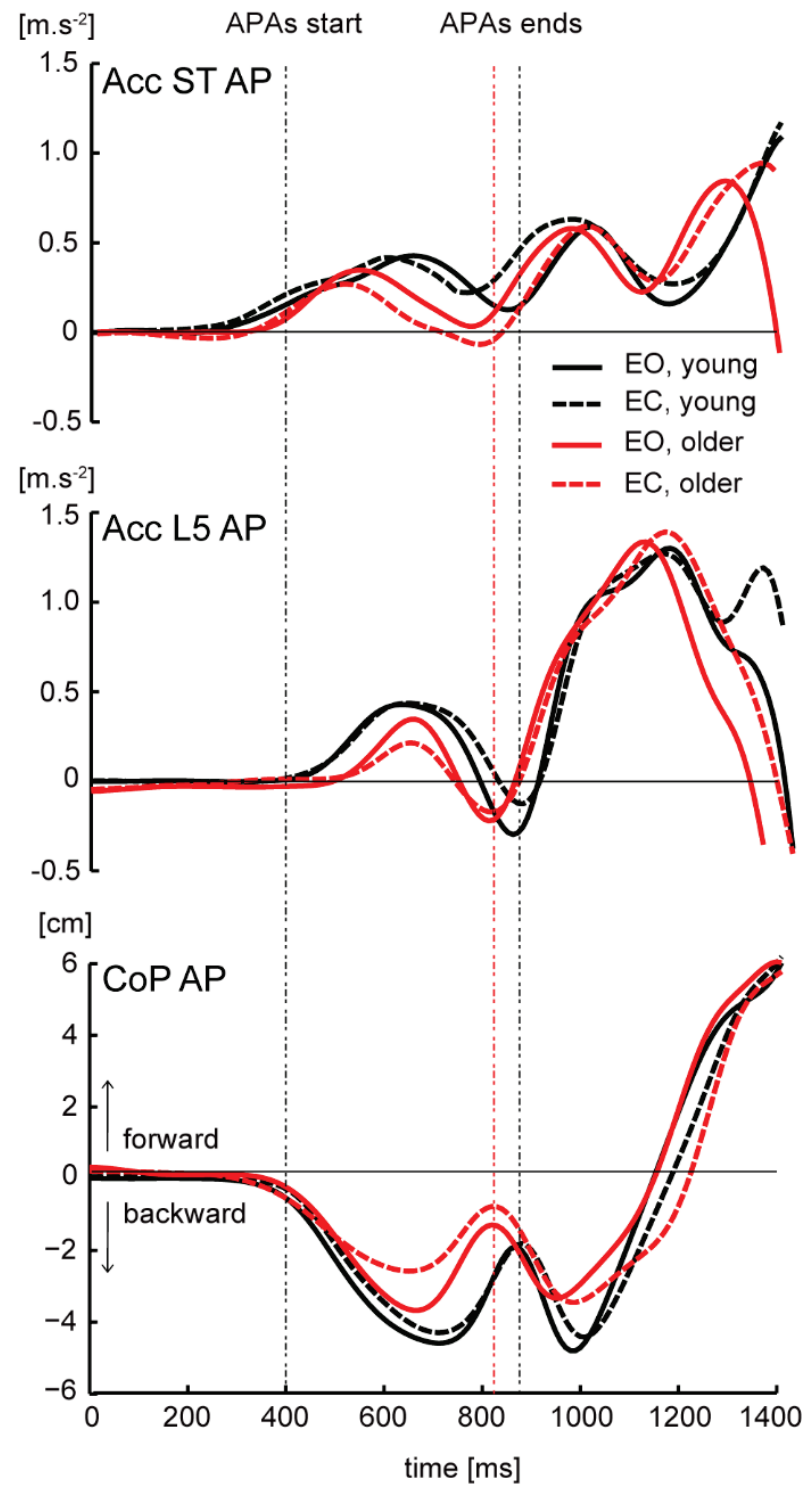

Fig. 2. Averaged excursions of center of pressure (CoP) displacement, and accelerations of lower (Acc L5) and upper (Acc ST) trunk in anterior-posterior (AP) direction during gait initiation in one representative young (black) and older (red) subject. Solid lines represent stepping with eyes open (EO), dashed lines represent stepping with eyes closed (EC). Dot-dashed lines determine approximated start and end of the anticipatory postural adjustments (APAs).

Comparison of gait initiation parameters further revealed significant differences between young and older adults. During stepping with eyes closed, they differed in all evaluated parameters (Fig. 1). Older adults demonstrated significantly decreased amplitudes of APAs (CoP, Acc L5, Acc ST) in AP direction as well as significantly shorter and slower first step than young adults during EC stepping. Moreover, older adults showed significantly smaller backward CoP peak and shorter first step with decreased max. velocity compared to young adults also during EO stepping. Besides the significant decrease of $\mathrm{CoP}$ and accelerations of upper and lower trunk in AP direction, also lateral CoP displacement (CoP ML) was significantly reduced in older adults during EO (3.58 \pm 0.26 vs. $4.52 \pm 0.27 \mathrm{~cm}$, $p<0.05$, older vs. young); and EC stepping (3.49 \pm 0.22 vs. $4.54 \pm 0.28 \mathrm{~cm}, p<0.01$, older vs. young).

\section{Discussion}

The transition from standing to steady state gait is a challenging daily activity, providing an ideal task to examine changes as a function of advancing age. The current study investigated whether anticipatory postural adjustments of gait initiation and kinematics of the first step are modified with absence of vision in relation to age. Our results showed that visual deprivation led to significant reduction of forward trunk accelerations during the anticipatory phase of stepping in older participants. Moreover, they significantly reduced first step length and max. velocity as well as prolonged duration of the first step while stepping with eyes closed. Contrary, young adults did not respond to absence of vision during stepping by significant changes of neither APAs amplitudes, nor first step kinematics. The results supported our first hypothesis that older adults would adopt a cautious stepping pattern when visual input is deprived as evidenced by reduced anticipatory postural adjustments together with modified first step kinematics. By adaptively modifying APAs and initial step, older adults might thereby reduce the dynamic degrees of freedom (Hallemans et al. 2009a). This could also cause a semi-rigid kinematic state, which increases stability under conditions of sensory deprivation and makes the stepping easier to complete, thus having more time for haptic exploration, and lowering the possibility of balance losses (Berard and Vallis 2006).

Previous study, however focused on walking, reported similar findings that older adults decreased walking velocity in EC condition (Cromwell et al. 2002). In agreement with our results, they also reported the shortening of step length which indicates that older adults minimized swing phase to reduce imbalance and increase stability of walking. Shortening of the step length is typical of cautious gait and it is likely that this enhances haptic exploration of the ground by the feet (Hallemans et al. 2010). In the study of Cromwell et al. (2002), changes in step length were paralleled by changes in upper body movements manifested as age-related decreases in the maximum predominant frequency of 
trunk movement. Our results enlarge the previous findings showing that reduced accelerations of upper and lower trunk were present in older subjects not even during walking, but already during the anticipatory phase of step initiation with visual input deprived. Therefore, in the absence of vision, older adults anticipated the alteration of their postural system and maximized stability in an effort to create a stepping pattern more resistant to alteration. Furthermore they relied mainly on vision and could not easily adapt when vision was removed. Based on our results, we indicate that gait initiation is strongly associated with increased reliance on visual input in older adults. Several factors can contribute to this. First, the sensorimotor mechanisms which control the dynamic stability may be less effective in older adults possibly due to age-related decreases in the proprioceptive (Zhou et al. 2016) and vestibular (Smith 2016, Alberts et al. 2019) systems. While young adults were very likely able to compensate for the lack of vision by using other senses in order to regulate the anticipatory postural adjustments of stepping as well as kinematics of the first step, older adults showed cautious APAs and the first step performance during EC condition indicating higher reliance on vision. Second, the sensory reweighting process may be delayed in elderly when visual input is altered or visual cues are missing (Jeka et al. 2010). Ageing alters the relative accuracy of sensory signals, and, consequently, older adults rely more on vision than on vestibular or proprioceptive signals to control balance (Saftari and Kwon 2018). It was shown that shift in sensory reweighting was primarily due to an age-related increase of the noise of vestibular signals (Alberts et al. 2019).

In contrast with our results, some studies reported that ageing seems to have little effect on the capability to manage stability during walking with the absence of vision (Saucedo and Yang 2017, Reynard and Terrier 2015). However, differences exist between these two studies and the current study. First, these studies examined steady state gait while we paid attention to the initiation of walking and second, they performed treadmill walking instead of overground walking executed by participants in our study. Whereas it was shown that kinematics and kinetics of gait were significantly modified during treadmill walking compared to overground walking in elderly (Watt et al. 2010), we preferred overground gait in our study. The lack of age-related changes reported by Saucedo and Yang (2017) may be due to small sample size (10 young and
6 older adults). Moreover, they analyzed only one trial of EO and one trial of EC walking for each subject which may not be sufficient to comprehensively inspect the postural responses. In the study of Reynard and Terrier (2015), sample size was much bigger (100 participants in age 20-69 years), but there was no specific importance placed on age-related changes.

Traditionally, the anticipatory postural adjustments of gait initiation are explored and quantified by the ground reaction forces (Jonsson et al. 2007, Henriksson and Hirschfeld 2005) and/or by the center of pressure displacements (Lu et al. 2017a, Plate et al. 2016, Hansen et al. 2016). Recently it was shown that APAs can be detected also by the inertial sensors worn on the trunk and described by the means of trunk accelerations (Mancini et al. 2016, Mancini et al. 2009). In our study, significant Age by Vision interaction effect on APAs was detected only for trunk acceleration amplitudes, and not for CoP amplitudes, indicating that forward trunk accelerations during anticipatory phase of stepping may be more sensitive measure to detect age-related changes of APAs due to absent vision compared to traditionally observed CoP. As long as the withdrawal of visual information caused significant decrease of forward upper and lower trunk accelerations, the amplitude of CoP displacements did not significantly change with the absence of vision in older adults.

Our second hypothesis that APAs and execution of the first step would be compromised in older adults, regardless of the visual condition, was partially supported by the results. Specifically, older subjects showed significantly smaller backward and lateral peaks of CoP displacements, irrespective of vision available or deprived, compared to young adults. Furthermore, length and max. velocity of the first step were significantly smaller during both EO and EC stepping in older compared to young adults. Our findings are consistent with previous ones that older adults exhibited significantly smaller lateral CoP displacement (Lu et al. 2017a) as well as smaller posterior and medial-lateral ground reaction forces (Henriksson and Hirschfeld 2005) during gait initiation than young adults. Gradually decreasing anticipatory activation of ankle muscles accompanied by less anticipatory backward CoP shift were indicated as a compensatory strategy in the postural adjustment in elderly. Contrary, Plate et al. (2016) reported that APAs are unaffected by age. However, their finding does not quite reflect the age-related changes in terms of young vs. older adults, because they considered 
as young controls the adults with mean age $43.8 \pm 2.9$ years. Thus comparability of our results to this study is limited. Congruent with our results, older participants demonstrated shorter (Lu et al. 2017a, Muir et al. 2014, Henriksson and Hirschfeld 2005) and slower (Muir et al. 2014) first step than young adults. Decreased length and velocity of the first step may reflect lack of propulsion strength in older subjects, but also a protective adaptation to a perceived threat to stability, as the center of mass must be accelerated from a stationary state, and the relatively small base of support (Muir et al. 2014).

There are few limitations worth noting. First, participants were instructed to initiate gait in response to an acoustic tone which might cause some constraints in adjusting of the anticipatory postural responses. The APAs of self-initiated gait were found smaller in magnitude than APAs of sensory cued stepping (Lu et al. $2017 b$ ). Second limitation may be the low number of trials collected per subject ( 5 trials).

In conclusion, ageing led to significant changes in the anticipatory postural adjustments of gait initiation and kinematics of the first step when the visual information was deprived. Older adults anticipated the alteration of their postural system by lack of vision which was manifested by significant reduction of upper and lower trunk accelerations. Moreover, they significantly reduced first step length and max. velocity as well as prolonged duration of the first step while stepping with eyes closed. Our results further indicate that forward trunk accelerations during the anticipatory phase of stepping could be more sensitive for detection of age-related changes in APAs than usually examined CoP. This study could offer deeper insight into how the visual deprivation influences the balance during gait initiation and how age affects the postural responses to visual threat.

\section{Conflict of Interest}

There is no conflict of interest.

\section{Acknowledgements}

This work was supported by the Slovak grant agency VEGA No. 2/0104/19 and by the Slovak research and development agency under the contract No. APVV-160233 .

\section{References}

ALBERTS B, SELEN L, MEDENDORP W: Age-related reweighting of visual and vestibular cues for vertical perception. J Neurophysiol 121: 1279-1288, 2019. https://doi.org/10.1152/jn.00481.2018

BERARD JR, VALLIS LA: Characteristics of single and double obstacle avoidance strategies: a comparison between adults and children. Exp Brain Res 175: 21-31, 2006. https://doi.org/10.1007/s00221-006-0529-0

BOUISSET S, DO MC: Posture, dynamic stability, and voluntary movement. Neurophysiol Clin 38: 345-362, 2008. https://doi.org/10.1016/j.neucli.2008.10.001

CROMWELL RL, NEWTON RA, FORREST G: Influence of vision on head stabilization strategies in older adults during walking. J Gerontol 57A: M442-M448, 2002. https://doi.org/10.1093/gerona/57.7.M442

CUEVAS-TRISAN R: Balance problems and fall risks in the elderly. Phys Med Rehabil Clin N Am 28: 727-737, 2017. https://doi.org/10.1016/j.pmr.2017.06.006

HALLEMANS A, BECCU S, VAN LOOCK K, ORTIBUS E, TRUIJEN S, AERTS P: Visual deprivation leads to gait adaptations that are age- and context-specific: I. Step Time parameters. Gait Posture 30: 55-59, 2009 a. https://doi.org/10.1016/j.gaitpost.2009.02.018

HALLEMANS A, BECCU S, VAN LOOCK K, ORTIBUS E, TRUIJEN S, AERTS P: Visual deprivation leads to gait adaptations that are age- and context-specific: II. Kinematic parameters. Gait Posture 30: 307-311, $2009 \mathrm{~b}$. https://doi.org/10.1016/j.gaitpost.2009.05.017

HALLEMANS A, ORTIBUS E, MEIRE F, AERTS P: Low vision affects dynamic stability of gait. Gait Posture 32: 547-551, 2010. https://doi.org/10.1016/j.gaitpost.2010.07.018

HANSEN C, LARUE J, DO MC, LATASH ML: Postural preparation to stepping: Coupled centre of pressure shifts in the anterior-posterior and medio-lateral directions. J Hum Kinet 54: 5-14, 2016. https://doi.org/10.1515/hukin$\underline{2016-0030}$

HENRIKSSON M, HIRSCHFELD H: Physically active older adults display alterations in gait initiation. Gait Posture 21: 289-296, 2005. https://doi.org/10.1016/j.gaitpost.2004.03.001 
HIRJAKOVA Z, NEUMANNOVA K, KIMIJANOVA J, SUTTOVA K, JANURA M, HLAVACKA F: Breathing changes accompanying balance improvement during biofeedback. Neurosci Lett 651: 30-35, 2017. https://doi.org/10.1016/j.neulet.2017.04.051

IOSA M, FUSCO A, MORONE G, PAOLUCCI S: Effects of visual deprivation on gait dynamic stability. ScientificWorldJournal 2012: Article ID 974560, 2012. https://doi.org/10.1100/2012/974560

JAHN K, STRUPP M, SCHNEIDER E, DIETERICH M, BRANDT T: Visually induced gait deviations during different locomotion speeds. Exp Brain Res 141: 370-374, 2001. https://doi.org/10.1007/s002210100884

JEKA KK, ALLISON LK, KIEMEL T: The dynamics of visual reweighting in healthy and fall-prone older adults. J Mot Behav 42: 197-208, 2010. https://doi.org/10.1080/00222895.2010.481693

JIAN Y, WINTER DA, ISHAC MG, GILCHRIST L: Trajectory of the body cog and cop during initiation and termination of gait. Gait Posture 1: 9-22, 1993. https://doi.org/10.1016/0966-6362(93)90038-3

JONSSON E, HENRIKSSON M, HIRSCHFELD H: Age-related differences in postural adjustments in connection with different tasks involving weight transfer while standing. Gait Posture 26: 508-515, 2007. https://doi.org/10.1016/j.gaitpost.2006.11.206

KANEKAR N, ARUIN AS: Aging and balance control in response to external perturbations: role of anticipatory and compensatory postural mechanisms. Age 36: 1067-1077, 2014. https://doi.org/10.1007/s11357-014-9621-8

LU C, AMUNDSEN HUFFMASTER SL, HARVEY JC, MACKINNON CD: Anticipatory postural adjustment patterns during gait initiation across the adult lifespan. Gait Posture 57: 182-187, $2017 \mathrm{a}$. https://doi.org/10.1016/j.gaitpost.2017.06.010

LU C, AMUNDSEN HUFFMASTER SL, TUITE PJ, VACHON JM, MACKINNON CD: Effect of cue timing and modality on gait initiation in Parkinson disease with freezing of gait. Arch Phys Med Rehabil 98: 1291-1299, 2017b. https://doi.org/10.1016/j.apmr.2017.01.009

MANCINI M, ZAMPIERI C, CARSLON-KUHTA P, CHIARI L, HORAK FB: Anticipatory postural adjustments prior to step initiation are hypometric in untreated Parkinson's disease: an accelerometer-based approach. Eur J Neurol16: 1028-1034, 2009. https://doi.org/10.1111/j.1468-1331.2009.02641.x

MANCINI M, CHIARI L, HOLMSTROM L, SALARIAN A, HORAK FB: Validity and reliability of an IMU-based method to detect APAs prior to gait initiation. Gait Posture 43: 125-131, 2016. https://doi.org/10.1016/j.gaitpost.2015.08.015

MUIR BC, RIETDYK S, HADDAD JM: Gait initiation: The first four steps in adults aged 20-25 years, 65-79 years, and 80-91 years. Gait Posture 39: 490-494, 2014. https://doi.org/10.1016/j.gaitpost.2013.08.037

O'MALLEY MJ: Normalization of temporal-distance parameters in pediatric gait. J Biomech 29: 619-625, 1996. https://doi.org/10.1016/0021-9290(95)00088-7

PATLA AE: Understanding the roles of vision in the control of human locomotion. Gait Posture 5: 54-69, 1997. https://doi.org/10.1016/S0966-6362(96)01109-5

PLATE A, KLEIN K, PELYKH O, SINGH A, BÖTZEL K: Anticipatory postural adjustments are unaffected by age and are not absent in patients with the freezing of gait phenomenon. Exp Brain Res 234: 2609-2618, 2016. https://doi.org/10.1007/s00221-016-4665-x

REYNARD F, TERRIER P: Role of visual input in the control of dynamic balance: variability and instability of gait in treadmill walking while blindfolded. Exp Brain Res 233: 1031-1040, 2015. https://doi.org/10.1007/s00221-014$\underline{4177-5}$

RIETDYK S, RHEA CK: Control of adaptive locomotion: effect of visual obstruction and visual cues in the environment. Exp Brain Res 169: 272-278, 2006. https://doi.org/10.1007/s00221-005-0345-y

ROCCHI L, CHIARI L, MANCINI M, CARLSON-KUHTA P, GROSS A, HORAK FB: Step initiation in Parkinson's disease: Influence of initial stance conditions. Neurosci Lett 406: 128-132, 2006. https://doi.org/10.1016/j.neulet.2006.07.027

RUBENSTEIN L: Falls in older people: epidemiology, risk factors and strategies for prevention. Age Aging 35: 37-41, 2006. https://doi.org/10.1093/ageing/afl084

SAFTARI LN, KWON OS: Ageing vision and falls: A review. J Physiol Anthropol 37: $11,2018$. https://doi.org/10.1186/s40101-018-0170-1 
SAUCEDO F, YANG F: Effects of visual deprivation on stability among young and older adults during treadmill walking. Gait Posture 54: 106-111, 2017. https://doi.org/10.1016/j.gaitpost.2017.03.001

SMITH PF: Age-related neurochemical changes in the vestibular nuclei. Front Neurol 7: 20, 2016. https://doi.org/10.3389/fneur.2016.00020

TERRIER P, REYNARD F: Effect of age on the variability and stability of gait: a cross-sectional treadmill study in healthy individuals between 20 and 69 years of age. Gait Posture 41: 170-174, 2015. https://doi.org/10.1016/j.gaitpost.2014.09.024

VAN MELICK N, MEDDELER BM, HOOGEBOOM TJ, NIJHUIS-VAN DER SANDEN MWG, VAN CINGEL REH: How to determine leg dominance: The agreement between self-reported and observed performance in healthy adults. PLoS One12: e0189876, 2017. https://doi.org/10.1371/journal.pone.0189876

WATT RJ, FRANZ JR, JACKSON K, DICHARRY J, RILEY PO, KERRIGAN DC: A three-dimensional kinematic and kinetic comparison of overground and treadmill walking in healthy elderly subjects. Clin Biomech 25: 444-449, 2010. https://doi.org/10.1016/j.clinbiomech.2009.09.002

WINTER DA: A.B.C (Anatomy, Biomechanics, Control) of Balance during Standing and Walking. Waterloo Biomechanics, Waterloo, 1995, $56 \mathrm{p}$.

WUEHR M, SCHNIEPP R, PRADHAN C, ILMBERGER J, STRUPP M, BRANDT T, JAHN K: Differential effects of absent visual feedback control on gait variability during different locomotion speeds. Exp Brain Res 224: 287-294, 2013. https://doi.org/10.1007/s00221-012-3310-6

ZHOU J, LIPSITZ L, HABTEMARIAM D, MANOR B: Sub-sensory vibratory noise augments the physiologic complexity of postural control in older adults. J Neuroeng Rehabil 13: 44, 2016. https://doi.org/10.1186/s12984-016-0152-7 